\title{
Zur Theorie der Verhaltensgeographie
}

Jegliches Nachdenken, sei es wissenschaftlicher oder vorwissenschaftlicher Art, über die Bedingungen menschlichen Verhaltens hat schon immer «Theorie» irgendeiner Form bzw. irgendeines Inhalts mit eingeschlossen. So bezeugen selbst naiv empiristische Sichtweisen, wie sie etwa in der traditionellen Geographie auftreten, (wenngleich verdeckt) hohen «theoretischen» Gehalt in dem Sinne, daß sie mit vorgeprägten Bildern (z. B. vom Menschen als «Landschaftsgestalter»), mit sanktionierten Untersuchungsperspektiven (z. B. der "physiognomischen Betrachtungsweise»), mit schematisierten Darstellungsformen (z. B. dem «länderkundlichen Prinzip») u. ä. arbeiten. Solchen Sichtweisen stehen Ansätze wie die moderne geographische Verhaltensforschung gegenüber, welche sich entschieden für die Notwendigkeit eines (offenen) Theorieeinsatzes aussprechen, und in denen demzufolge der theoretische Gehalt im allgemeinen wenig strittig ist.

Mir geht es im folgenden jedoch nicht primär um den Nachweis eines mehr oder minder deutlichen Theoriebezugs in geographischen Forschungsansätzen, speziell hier: in der Verhaltensgeographie. Wir können vielmehr davon ausgehen, daß ein solcher Bezug besteht und zugestanden wird, so daß wir uns gleich dem Charakter entsprechender Theorien zuwenden, m.a.W. der Frage nachgehen können, was durch diese (mehr oder weniger umfassenden) FrageAntwort-Komplexe hervorgehoben, in Zusammenhang gebracht, beurteilt, bewertet, ausgeblendet oder verstellt wird. Es geht also letztlich um die pragmatische Dimension, insbesondere um die gesellschaftlich-politische Bedeutung solcher wissenschaftlichen Konstrukte zur Erfassung, Erklärung und Planung der Realität. Dabei stehen wir allerdings vor der Schwierigkeit, daß uns in der Verhaltensgeographie - und dies gilt auch für Teile der anderen Verhaltenswissenschaften - ausgearbeitete Theorien kaum vorliegen. Was uns in der Regel vorliegt, sind Forschungsansätze mit einander relativ lose zugeordneten Leitfragen, Begriffen, Hypothesen, Methoden und Techniken, also Konstrukte, die man am besten als "conceptual frameworks» bezeichnen würde.

Ich werde mich außerdem in meinem Referat auf die Theorie der jüngeren geographischen Verhaltensforschung beschränken. Menschliches Verhalten ist ja in der Geographie schon seit jeher thematisiert worden, insbesondere im Rahmen geodeterministischer und possibilistischer landschafts- und länderkundlicher Forschungsperspektiven, u.z. als natur- oder umweltabhängiges/-unabhängiges Reagieren bzw. als natur- oder kulturlandschaftsgestaltendes/-umgestaltendes Handeln. Dennoch wurde in dieser «klassischen» Phase der Anthropogeographie das Verhalten nicht eigentlich zum Gegenstand der Untersuchung gemacht (weshalb in der Regel diese Ansätze auch nicht direkt zur Verhaltensgeographie gerechnet werden). Ein solches Forschungsziel taucht erst in der jüngsten Phase der Entwicklung der Anthropogeographie auf '). Untersucht wird jetzt das (räumliche) Verhalten selbst, und mithin werden die («raumbezogenen») Einstellungen und das («raumbedingte») Agieren von in Gesellschaft lebenden Menschen analysiert. Diese jüngere geographische Verhaltensforschung zeichnet sich nicht nur auf der inhaltlich-thematischen Ebene durch einen bewußten (d. h. gezielten) Einsatz von (Verhaltens-)Theorien aus, sondern ist auch auf der formal-methodischen Ebene mit neueren Wissenschaftsauffassungen, insbesondere der analytisch-nomologischen Richtung, verbunden. In der Tendenz erhält die Geographie damit zum ersten Mal Anschluß an eine überdisziplinäre Entwicklung und an die «moderne» Sozialwissenschaft.

Wenn wir bei den folgenden Betrachtungen ${ }^{2)}$ also von der Voraussetzung ausgehen können, daß die jüngere geographische Verhaltensforschung "modern» im oben genannten Sinn ist, wäre dann noch zu fragen, an welchem Grundproblem eine solche Verhaltensgeographie orientiert ist. Nun: Ausgegangen wird von der Beobachtung, daß verschiedene Menschen dieselben (Umwelt-)Gegebenheiten unterschiedlich bewußtseinsmäßig wahrnehmen bzw. auf diese unterschiedlich handelnd reagieren. Aus dieser grundsätzlichen Feststellung resultieren zwei im allgemeinen getrennte - Forschungsansätze, nämlich ein i.e.S. verhaltenstheoretischer (oder «behavioristischer», auch: "am Einstellungs-Konzept orientierter») und ein i.e.S. handlungstheoretischer (oder «aktionistischer», auch: «entschei-

Dr. Günther Beck, Geographisches Institut der Universität Göttingen, Goldschmidtstraße 5. D-3400 Göttingen. 
dungstheoretischer») Ansatz ${ }^{3)}$. Der erste Ansatz beinhaltet Untersuchungen über die Wahrnehmung von Objekten, Ereignissen und Situationen der Umwelt, denen gegenüber der Mensch Gefühle empfindet, und auf die er sein zweckbestimmtes Handeln ausrichtet ${ }^{4}$. Die Erforschung solcher raumbezogener Einstellungen, solchen aktivitätsneutralen Verhaltens gegenüber dem Raum $^{5)}$ geschieht überwiegend durch Befragung der «Meinung» (als verbaler und/oder sensomotorischer Äußerung) der Menschen und verlangt in der Hauptsache den Einsatz psychologischer Erkenntnisse, Methoden und Techniken. Die bei der Einstellungsforschung auftretenden Schwierigkeiten versucht der zweite Ansatz von vornherein dadurch zu umgehen, daß nicht mehr vorrangig der «Mentalbereich» zum Gegenstand von Untersuchungen über menschliches Verhalten gemacht wird; sondern diese konzentrieren sich primär auf die «letzten Glieder in der verhaltensgesteuerten Handlungskette räumlicher Aktivitäten»6), die Analysen zielen mehr auf das raumbedingte Agieren, auf das offenkundige Verhalten im Raum ${ }^{7}$ ). Der am Einstellungskonzept orientierte Wissenschaftler sucht also die steuernden Faktoren menschlichen Agierens und Reagierens eher auf der Seite der «subjektiven" Vorbedingungen des Verhaltens, in der Organisationsstruktur und dem Normenpotential des Bewußtseins, und im Gefolge davon in den mental repräsentierten politischen/sozialen Status- und Rollen-Bedingungen von individuellem Verhalten. Dagegen vermutet der aktionstheoretisch argumentierende Forscher jene das menschliche Handeln bestimmenden Faktoren eher auf der «objektiven» Seite, d. h. im Feld der außerhalb des Bewußtseins liegenden Realisationsbedingungen des Verhaltens zu finden. Dementsprechend konzentriert sich dieser Ansatz mehr auf das Studium der direkt zu beobachtenden Orientierungsprozesse und Entscheidungsabläufe, Aktivitätsradien und Bewegungsrichtungen, und im Gefolge davon auf die äußeren, materiellen/ökonomischen Handlungszwänge und Handlungsmöglichkeiten.

Das Gesagte läßt sich am besten anhand von entsprechenden Beispielen aus der geographischen Forschungspraxis verdeutlichen.

1. Betrachten wir zuerst J. WOLPERTs «Räumliche Analyse des Entscheidungsverhaltens in der mittelschwedischen Landwirtschaft» (1970). In dieser Untersuchung geht der Autor von der Feststellung aus, daß die Bauern in Mittelschweden unterschiedliche (räumlich variierende) Arbeitseinkommen realisieren. Er führt diese Einkommensunterschiede - bzw. genauer: die Lücke zwischen dem einem Betrieb möglichen und dem tatsächlichen Einkommen - auf ein mehr oder weniger hohes "Anspruchsniveau» der einzelnen Landwirte zurück. Offensichtlich sitzen die Landwirte mit weniger hohem Anspruchsni- veau dort, wo größere Abweichungen, diejenigen mit höherem Anspruchsniveau dagegen dort, wo geringere Abweichungen vom möglichen Optimum der Fall sind. Die unterschiedlichen Ergebnisse bei der Realisierung von Arbeitseinkommen werden also von WOLPERT aus unterschiedlichen individuellen (und regional differenzierten) Einstellungen zum optimalen Erfolg erklärt.

2. In aktionsräumlichen Untersuchungen, z. B. in S. MÅRTENSSONs Studie über den «Einfluß regionaler Disparitäten auf die Zeitbudgets ausgewählter Haushaltstypen» (1979), werden nicht Einstellungsunterschiede gegenüber der Umwelt, sondern unterschiedliche Bewegungsspielräume in dieser analysiert. So wird die Beobachtung gemacht, daß es in jeder Region Menschen mit unterschiedlichen Möglichkeiten gibt, Distanzen zu überwinden. Zum einen wird dies der Struktur der Umwelt, etwa der Verteilung der zentralen Orte und ihrer Verbindung untereinander, zugeschrieben. Zum andern wird es in Zusammenhang gebracht mit den einer einzelnen «Entscheidungseinheit» zur Verfügung stehenden «Ressourcen» (Zeit, Geld usw.). Ein spezielles Hilfsmittel, Distanzen zu überwinden, stellt z. B. das Auto dar, das in vielen aktionsräumlichen Untersuchungen eine gewichtige Rolle spielt. Der Aktionsraumforscher kann feststellen, daß mit zunehmender Entfernung von einem zentralen Einkaufsort die Gruppe der Autobesitzer die Gruppe der NichtAutobesitzer unter den Konsumenten übertrifft. An diese Feststellung anschließend erfolgen in der Regel weitere Differenzierungen der Käufer nach Alter, Familienstand, Sozialstatus usw. beziehungsweise des Käuferverhaltens nach Einkaufshäufigkeit, -dauer, -summen usw. ${ }^{8)}$

Die den beiden Ansätzen gemeinsame Frage nach den Bedingungen räumlichen Verhaltens wird also unterschiedlich zu beantworten versucht. Die i.e.S. verhaltenstheoretische Geographie unternimmt es, bestimmte gesellschaftliche Phänomene wie das Handeln der Menschen selbst oder die Ergebnisse dieses Handelns mit den Absichten und Beweggründen (wie dem "Anspruchsniveau») der Menschen in Verbindung zu bringen. Diese Art der Erklärung menschlichen Verhaltens leidet jedoch unter der prinzipiellen Schwierigkeit, daß es praktisch unmöglich ist, jemals mit Sicherheit festzustellen, welches die tatsächlichen Motive waren, die ein bestimmtes Geschehen bewirkt haben. Man kann folglich das, was sich ereignet hat, stets dadurch «erklären», daß man sagt, gewisse Menschen hätten mit gewissen Absichten gehandelt, womit man offensichtlich alles erklären kann'). Selbst da, wo der Verhaltensforscher bestimmte Vorstellungsinhalte, wie z. B. mental maps, mit einiger Genauigkeit erfragen kann, steht er vor dem Problem, daß damit in bezug auf die Erklärung tatsächlichen Verhaltens kaum etwas ge- 
wonnen ist. Eine mental map ist eben (zunächst) nur das Bild von einer mental map ${ }^{10)}$.

Dem offenkundigen Zirkelschluß, z. B. unterschiedliche Einkommen mit unterschiedlichen Anspruchsniveaus wechselweise «erklärend» zu verbinden, scheint also der behavioristische Ansatz ganz allgemein schwerlich zu entkommen. Selbst dann, wenn solche Persönlichkeitsvariablen wie das "Anspruchsniveau» nicht als dem Individuum innewohnende Entitäten angesehen werden, sondern in andere Variablen, z. B. in sog. Grundbedürfnisse wie «Realanpassung», «Leistung», «Erfolgssicherung» usw. zerlegt werden ${ }^{11)}$, wird das Problem nur verschoben, d.h. der Zirkelschluß nicht aufgehoben. Die häufigste und bedeutsamste Form solcher Tautologien liegt dann vor, wenn etwa aus einem beobachtbaren Verhalten in einer speziellen oder allgemeinen Leistungssituation auf ein «Leistungsbedürfnis» geschlossen wird. In diesem Fall wird ja lediglich die Ausgangsfeststellung in einfacher Umformulierung wiederholt; $d$. h. es erfolgt eine sprachliche Abwandlung, allerdings unter ideologieverdächtiger Bezugnahme auf bestimmte quasi-anthropologische «Einstellungen» des Menschen. So wird auch in keiner Weise verständlich (gemacht), weshalb gerade diese «Hauptmotive» (wie die genannten "Grundbedürfnisse») und keine anderen in den verhaltenstheoretischen Definitionen, Hypothesen oder Theorien auftreten ${ }^{12)}$.

Der aktionistische Ansatz in der Geographie scheint nun die positive Wendung gegenüber den logischen Ungereimtheiten und den ideologischen Implikationen, die der behavioristische Ansatz aufbaut, zu garantieren. Gegenstand der Untersuchung ist jetzt die Reaktion der kleinsten Entscheidungseinheiten (Individuen, Haushalte, Betriebe, Institutionen) auf die sozialräumliche Situation, oder - wie HÄGERSTRAND es ausdrückt ${ }^{13}$ ) - auf die "Angebotsstruktur» der Umwelt, insbesondere auf technische u.a. Innovationen. Der Ansatz verfällt dabei nicht dem (Geo-)Determinismus, menschliches Verhalten als direkten Ausfluß solcher Umweltgegebenheiten zu begreifen. Sondern für diesen Ansatz ist die Annahme konstitutiv, daß das tatsächliche (räumliche) Verhalten vermittelt wird über Entscheidungsprozesse, durch welche die Bewertung der Umweltgegebenheiten mit den zur Verfügung stehenden Ressourcen verbunden wird. Der Aktionsraumforscher beansprucht also nicht mehr, das Verhalten der Menschen aus allgemeinen Persönlichkeitsvariablen zu erklären. «Erklären» von Verhalten heißt bei ihm, dieses auf die Reaktion auf unmittelbare, situative Bedingungen (nämlich vorfindliche Umweltstrukturen und vorhandene Bewertungsmuster) zurückzuführen, die nunmehr als Bedingungen von Verhalten schlechthin erscheinen. Damit wird abstrahiert von jenen Verhältnissen, die diese Umweltstrukturen und Bewertungsmuster selbst wieder be- stimmen, und die den Rahmen ihrer Entstehung, Ausprägung und Veränderung vorzeichnen: die Bedingungen der gesellschaftlichen (Produktions-)Verhältnisse ${ }^{14)}$.

Insofern gleichen sich die beiden Teilansätze geographischer Verhaltensforschung. Jedoch ist ein zweifacher Vorzug des aktionistischen vor dem behavioristischen Ansatz zu vermerken: Indem die Bestimmungsgründe für das Verhalten von Menschen primär nicht mehr im «subjektiven» Bereich des menschlichen Bewußtseins gesucht werden, wird es zum einen möglich, Verhalten nicht nur von Individuen, sondern auch von anderen Entscheidungseinheiten, nämlich von Haushalten, Betrieben, Institutionen, zum Gegenstand von Verhaltensforschungen zu machen. Zum andern liegt sicherlich in der Erfassung der «objektiven», außerhalb des Bewußtseins liegenden Bedingungen des Verhaltens die Möglichkeit einer kritischen Überschreitung behavioristischer und aktionistischer Forschungen. $\mathrm{Da} ß$ eine solche «Überschreitung» allerdings «verhaltenswissenschaftlich» nicht zu leisten ist, soll im folgenden einsichtig gemacht werden. Dafür ist jedoch noch eine weitere Durchleuchtung der verhaltenswissenschaftlichen Theorie erforderlich.

Einen integrierenden Bestandteil verhaltenswissenschaftlicher Theorie bilden Annahmen über notwendig-allgemeine Zusammenhänge zwischen den Bedingungen und den Effekten menschlichen Verhaltens. Diesbezügliche «gesetzmäßige» Feststellungen lassen sich als Kausalverknüpfungen allgemeiner Geltung begreifen und als Invarianzen im Handlungsvollzug einzelner oder Gruppen von Menschen darstellen. Beispiele für solche "Gesetze» sind - in bezug auf den behavioristischen Ansatz -: «Menschen verhalten sich entsprechend ihrem (individuell-persönlichen) Anspruchsniveau»; oder - in bezug auf den aktionistischen Ansatz -: "Menschliches Handeln wird bestimmt durch den Versuch, unter Einsatz der zur Verfügung stehenden Mittel (z. B. einem Auto) ein Maximum an Nutzen (z. B. an Bewegungsspielraum) zu erreichen».

Wie werden nun solche allgemeinen Erkenntnisse regelhaften (Raum-)Verhaltens, die als "nomologische Hypothesen» begriffen werden, gewonnen? Das Verfahren, das dem Verhaltensforscher dazu dient, auf derartige Gesetzmäßigkeiten zu kommen, läßt sich kurz etwa folgendermaßen beschreiben: Ausgehend von Einzelfeststellungen über Zustände und Ereignisse, die das (räumliche) Handeln von Menschen betreffen, wird im Zuge der Verallgemeinerung solcher Feststellungen nicht nur von den individuell-besonderen Bedingungen - den «Randbedingungen» - wie etwa Alter, Familienverhältnisse, Bildungsstand, Informiertheitsgrad usw. abgesehen, sondern ebenfalls von den gesellschaftlichallgemeinen Bedingungen - den «Rahmenbedingun- 
gen» - der Handlungssituation wie etwa den ökonomischen und politischen Herrschafts- und Machtstrukturen, den sozialen Normengefügen usw. abstrahiert. Die konkret-historischen Verhältnisse, unter denen sich die betreffenden Menschen verhalten, werden zwar auf der Ebene der empirischen Einzelfeststellungen noch benannt; in der Theorie über menschliches Verhalten aber erscheinen diese Verhältnisse nicht mehr, sie sind für die Theorie zufällig, sie werden zu «Rahmen-» oder «Randbedingungen» gemacht bzw. als «Störfaktoren» in bezug auf die Geltung der überhistorisch-abstrakten nomologischen Hypothese hingestellt. Dazu ein Beispiel: Ein jugendlicher Arbeiter in der BRD, seit einem Jahr arbeitslos, zeigt kein Interesse, einen Arbeitsplatz in einem $40 \mathrm{~km}$ entfernten privaten Großbetrieb anzunehmen, sondern «begnügt» sich mit Gelegenheitsarbeiten in der Kleinstadt, in der er mit seiner Freundin wohnt... - Es dürfte nicht schwer fallen, das Verhalten dieses Jugendlichen mit einer der beiden oben aufgeführten nomologischen Hypothesen oder einer anderen entsprechenden Gesetzeshypothese zu «erklären». Dies würde der Verhaltenswissenschaftler dadurch bewerkstelligen, daß er dies aber gar nicht tun würde, sondern daß er nun doch die konkreten Rahmen- und Randbedingungen zur Erklärung des Verhaltens dieses Jugendlichen heranzöge ${ }^{15)}$. Ersichtlich tritt dabei die «Theorie» bzw. die ihr zuzurechnende nomologische Hypothese völlig zurück, sie ist entbehrlich, hat allenfalls legitimatorischen Wert (letzteres bedeutet vom wissenschaftslogischen Aspekt her sehr wenig, vom wissenschaftspraktischen Aspekt her aber sehr viel). Die prinzipielle Überflüssigkeit der Theorie bzw. der hypothetischen Gesetzesaussage drückt sich auch dadurch aus, daß sie beliebig durch andere derartige gedankliche Konstrukte ersetzt werden kann.

Die Abstraktion von den konkret-historischen gesellschaftlichen Verhältnissen führt nicht nur bei beiden Ansätzen zu falschen Verallgemeinerungen, indem beide Male das Besondere, nämlich das eine Mal ein habitualisiertes Verhalten (z. B. das Anspruchsniveau), das andere Mal eine regulierende Handlungsbedingung (z.B. eine Ressource), zum Allgemeinen erhoben wird; sondern eine derartige Abstraktion gestattet bzw. erzwingt darüber hinaus eine Anthropologisierung des Menschenbildes. Der Verhaltensforscher kann bzw. muß sich ein von allen «störenden Faktoren» gereinigtes «Bild» vom Menschen schaffen, da er den Menschen nicht mehr als wirklichkeitsbestimmtes, geschichtliches Wesen erfaßt. Ausdruck solcher «Reinigung» (HOLZKAMP) sind Vorstellungen vom Menschen wie diejenige vom "Satisficer», vom Menschen, der sich damit «begnügt», sich gemäß seinem individuellen «Anspruchsniveau» zu verhalten; oder der Verhaltensforscher kommt zu Vorstellungen vom Menschen als «Spieler», der unter Einberechnung der ihm zur Ver- fügung stehenden Mittel seine Handlungsmöglichkeiten gegenüber der Umwelt abschätzt.

Ich werde auf die gesellschaftliche Funktion einer solchen Vorgehensweise noch zurückkommen. $\mathrm{Zu}$ nächst jedoch muß ich auf einen möglichen, nicht unberechtigten Einwand eingehen, nämlich auf die Frage, ob nicht jede Theorie von besonderen als auch von allgemeinen konkret-historischen Bedingungen des Verhaltens abstrahieren muß, denn sonst - so würde man sagen - wäre sie ja wohl nicht mehr «Theorie», sondern die "Welt» selbst. Nun zeichnet sich die hier kritisierte (Verhaltens-)Theorie allerdings nicht nur dadurch aus, daß sie von dieser oder jener Rahmen- und Randbedingung, sondern daß sie von diesen Bedingungen schlechthin abstrahiert. Und dies bedeutet, in der Konsequenz, auch die Abstraktion von einer immer als möglich anzunehmenden Systemstruktur dieser Rahmen- und Randbedingungen. Eine alternative Theorie, von der wir mit fortschreitender Kritik an den vorherrschenden Verhaltenstheorien zunehmend Vorstellung gewinnen, könnte sicherlich nicht alle Rahmen- und Randbedingungen in Form von begrifflich gefaßten Realabstraktionen in ihren Erklärungsansatz einbeziehen. Doch würde gerade die Annahme einer möglichen Systemstruktur zwischen bestimmten Rahmen- und Randbedingungen das Selektionskriterium dafür liefern, welche dieser Bedingungen notwendigerweise schon auf der Ebene der Theorie und nicht erst als die Allgemeinaussagen (bloß) "störende Faktoren» zu berücksichtigen wären.

Bleiben wir bei dieser im vorstehenden schon von der wissenschaftslogischen Seite her kritisierten «Störfaktorenmethode». In dieser Art des Vorgehens steckt ja überdies das metatheoretische Urteil, daß die Faktoren, von denen abstrahiert wurde, für die Erklärung des befragten Phänomens unwesentlich seien. Auf der metatheoretischen Ebene ist nicht einsichtig zu machen, weshalb der Verhaltensforscher in seinen Gesetzen z. B. von einem Allgemeinen wie der Klassenlage eines Menschen abstrahiert. (Man vergleiche diesbezüglich die oben angeführten oder andere nomologische Hypothesen.) - Man verstehe dies recht: Mit diesem Hinweis ist überhaupt noch nichts darüber ausgesagt, wie lohnend es für die Empirie wäre, als wesentlich Allgemeines in den Verhaltensbedingungen eines Menschen seine Klassenlage zu bestimmen und dies auf der Theorieebene zu berücksichtigen. - Verständlich wird der Charakter eines Ansatzes, welcher in der Theorie von der Klassenlage eines Menschen abstrahiert, dagegen erst, wenn man ihn ideologiekritisch betrachtet, nämlich seinen Bezug zur bürgerlichen Realität bestimmt. Dann wird deutlich, daß «sobald in der Theorie von den Klassen abstrahiert wird,... der Schein von Gleichheit (entsteht), die die bürgerliche Wirklichkeit und das falsche Bewußtsein von ihr charakteri- 
siert: die Gleichheit erscheint als Hauptsache, die ökonomische Ungleichheit als Nebensache» 16).

Das Normative, das in dieser Methodik der Abstraktion steckt, kann noch an einem anderen Sachverhalt deutlich gemacht werden. Häufig wird ja einem klassenanalytischen («marxistischen») Ansatz die Qualität zugeschrieben, er ziele auf die «Veränderung der bestehenden Verhältnisse», beispielsweise über die Veränderung des Verhaltens der Menschen. Diese Aussage (welche meist den Charakter eines Vorwurfs hat) trifft indes den Sachverhalt nur halb richtig. Denn dieses Ziel hat auch die nicht-klassenanalytisch argumentierende («nicht-marxistische») Verhaltensforschung, und sie leistet dafür Tag für Tag große Arbeit, indem sie derartige Veränderungen beschreibt, erklärt, prognostiziert und in praktischer Anwendung betreibt. Nur: da bei ihr alle jene anderen Bedingungen als die gesellschaftlichen Klassenverhältnisse - wie z. B. Persönlichkeitsvariablen, zur Verfügung stehende Ressourcen - wesentlich Allgemeines sind, erfaßt und verändert sie «bloß» die individuellen oder die situativen Bedingungen des Verhaltens im Rahmen der bestehen bleibenden gesellschaftlichen Klassenverhältnisse. - In diesem Zusammenhang trägt übrigens der Einwand nichts aus, daß man ja zuerst empirisch feststellen müsse, ob überhaupt Klassenverhältnisse bestünden. Abgesehen von dem kruden Empirismus, man könne solche Verhältnisse «theorielos», mit leerem Bewußtsein, beobachten, besteht ja der Unterschied zwischen marxistischer und nicht-marxistischer («bürgerlicher») Theorie nicht darin, daß letztere keine «Klassen» kennt. Denn «die bürgerliche Theorie (hat) nie geleugnet, daß es Klassen, oder bürgerlich gesprochen, gesellschaftliche Ungleichheit gibt. Die Annahme bewußter Fälschung ist viel zu plump. Der Witz der bürgerlichen Theorie besteht nicht in der Leugnung der ökonomischen Ungleichheit, wie soll das auch möglich sein, sondern in der Abstraktion von ihr. Sie gilt als bloß empirisches Randphänomen, das in der Theorie vernachlässigt werden kann»17).

Die Feststellung, daß die Methode, welche ein solches Vorgehen absichert, nämlich die «Störfaktorenmethode», nicht funktionslos ist, nährt den Verdacht, daß das eigentliche Ziel des Verhaltensforschers gar nicht die Theorie (bzw. die Erklärung) ist. Was aber ist dann sein Ziel? Werfen wir noch einmal einen Blick darauf, vor welchem Problem der Verhaltensforscher bei der Aufstellung allgemeiner Regelmäßigkeiten im Verhalten von Menschen steht. Auf der Suche nach solchen Regelmäßigkeiten sieht er sich einem prinzipiellen Dilemma gegenüber: $\mathrm{Er}$ stößt sehr bald auf das Problem, daß immergleiches (räumliches) Verhalten von Menschen empirisch nicht zu beobachten ist - und zwar selbst nicht unter als gleich angesehenen allgemeinen (Rahmen-)Bedingungen oder unmittelbaren (Rand-)Bedingungen des Verhaltens. An diesem Tatbestand ändert auch eine Verfeinerung der Untersuchungsmethoden bzw. der Meßverfahren nichts.

Damit befindet sich der Verhaltensforscher in der gleichen Lage wie der Physiker, welcher feststellen muß, daß ein Gegenstand (z.B. ein Stein) nie gleich fällt, nie gleich fällt wie ein anderer Gegenstand (ein anderer Stein, eine Vogelfeder), und der dennoch zur Formulierung eines Fall-«Gesetzes» gelangt. Die Vorgehensweise des Naturwissenschaftlers ist ja hinreichend bekannt: Er abstrahiert bei der Aufstellung des Fallgesetzes von den Verhältnissen eines bestimmten Raumes, einer bestimmten Zeit und eines bestimmten Objekts. Die Frage ist, ob der Verhaltenswissenschaftler bei der Erforschung der «Gesetze» menschlichen Verhaltens nicht ebenso vorgehen kann, nicht ebenso vorgeht wie der Naturwissenschaftler. Nach den obigen Ausführungen müßte deutlich geworden sein, daß er dies tut.

Fragen wir uns, welches Interesse den Verhaltensforscher leiten könnte, auf diese Weise vorzugehen. Ziehen wir noch einmal die (modernen) Naturwissenschaften zu Rate. Von diesen wissen wir, daß sie «die Gesetze sentdeckt) (hatten), denen die Natur zu gehorchen schien. Sie hatten festgestellt, daß die Erscheinungen der Natur bestimmten Gesetzmäßigkeiten unterliegen, aufgrund deren Kenntnis sie, wie auch die Folgen von Eingriffen in ihren Ablauf, voraussagbar waren. Dadurch wurde es möglich, die Natur zu beherrschen. Was sollte dagegen sprechen, daß nicht nur die Natur (im Sinne der Physik», sondern auch die 〈Natur〉 des Menschen solchen Gesetzmäßigkeiten unterliegt?»18). Beruht also der gezielte Erkenntnisdrang des Verhaltensforschers für die Regelmäßigkeiten menschlichen Verhaltens nicht ebenfalls auf dem Interesse an dessen Voraussagbarkeit und Beherrschung (und nicht auf einem irgendwie gearteten Erkenntnisideal)? Dafür spricht, daß in bezug auf die Anwendung verhaltenswissenschaftlicher Untersuchungen vielfach sogar ausdrücklich das Interesse an der Voraussagbarkeit und Steuerbarkeit menschlichen Verhaltens als eigentlicher Anlaß und als eigentliches Ziel betont wird ${ }^{19}$ ).

Am besten läßt sich ein solches Interesse durch Umkehrschluß beweisen, und zwar aufgrund folgender Beobachtung: In dem Maße, wie das praktische Interesse an der Prognose und an der Beherrschung menschlichen Verhaltens Vorrang bekommt, verschiebt sich auch das Interesse an Theorie, deren prinzipielle Überflüssigkeit wir im Zusammenhang ihrer explikativen Funktion innerhalb des EmpirieTheorie-Verhältnisses schon aufgezeigt haben. Die Entbehrlichkeit der Theorie im Zusammenhang des Praxis-Theorie-Verhältnisses wird am deutlichsten beim aktionistischen Ansatz, welcher eine mögliche Theorie der bewußtseinsmäßigen Voraussetzungen menschlichen Handelns in die bekannten «Schwarzen Boxen» verbannen und darin belassen kann. Die 
Etablierung von «black-box»-Modellen kann geradezu als Ausdruck des Verzichts auf Theorie angesehen werden ${ }^{20)}$, abgesehen davon, daß einige Wissenschaftler subjektiv ehrlich an den «heuristischen Wert» dieser Schwarzen Boxen glauben. Die Praktiker (in den Industrieunternehmen, Schulen, Gefängnissen, Meinungsforschungsinstituten, Planungsgremien usw.) jedenfalls können auf «allgemeine Verhaltenstheorie» völlig verzichten ${ }^{21)}$. Damit wird deutlich, daß sich die Brauchbarkeit «empiristischer» Verhaltenstheorie (wie wir sie ab jetzt bezeichnen wollen) auf deren ideologische Funktion reduziert.

Eine damit einhergehende, weitere Konsequenz der oben beschriebenen methodischen Vorgehensweise ist das Aufgeben des Wahrheitsanspruchs der empiristischen (Verhaltens-)Theorie. Im Selbstverständnis des analytisch-nomologischen Wissenschaftstheoretikers bedeutete dies bisher, daß eine solche Theorie falsifizierbar sein müsse, ansonsten sie dem Dogmatismus-Verdacht verfalle. Nun erlaubt aber offensichtlich die beschriebene Störfaktorenmethode, daß eine solcherart gebildete nomologische Hypothese oder ganze Theorie - entgegen der landläufig verbreiteten wissenschaftstheoretischen Meinung - niemals falsifiziert, sondern höchstens ausgewechselt werden kann (was ja immer wieder geschieht, und was weniger mit wissenschaftsinternen, als viel mehr mit wissenschaftsexternen Gründen die es im Einzelfall zu benennen gilt - zu tun hat ${ }^{22)}$ ). Denn einer solchen Gesetzeshypothese oder Theorie widersprechende Befunde können ja immer auf «störende Umstände» zurückgeführt werden. Dagegen nicht auf die Auswechselbarkeit der Theorie zu setzen, sondern den Anspruch auf eine mögliche $h i$ storische Wahrheit zu verfolgen, ist so «dogmatisch», wie die gesellschaftlichen Verhältnisse es sind, die eine solche Theorie zu beschreiben und zu erklären versucht.

Die bisherigen Ausführungen sind - dies soll auch das zuletzt Gesagte deutlich machen - keineswegs als ein Plädoyer für den Verzicht auf jegliche Theorie über menschliches Verhalten aufzufassen. Nur kann die Alternative nicht eine zusätzliche (und «bessere») Verhaltenstheorie, sondern nur eine (nichtempiristische) Gesellschaftstheorie sein. Einige Elemente einer solchen "Gesellschaftstheorie», die als «kritische Negation» zu jeglicher «Verhaltenstheorie» aufzufassen wäre, möchte ich zum Schluß noch diskutieren, und zwar möglichst orientiert am konkreten Problem und nicht so sehr wissenschaftsmethodisch-allgemein.

Man könnte sich z. B. noch einmal die Frage stellen, ob es nicht doch so etwas wie ein (unterschiedliches) "Anspruchsniveau» bei menschlichen Individuen gibt. Dieses Problem erfährt im Zusammenhang der behavioristischen Theorie, wie aufgezeigt wurde, sicherlich eine falsche Behandlung: Das «An- spruchsniveau» erscheint da als ursprüngliche Persönlichkeitsvariable, als eine der Person innewohnende «anthropologische» Qualität; denn im behavioristischen Verständnis von "Anspruchsniveau» sind die objektiven Verhältnisse vollkommen in subjektive Verhaltensdispositionen aufgelöst.

Ein erster Schritt zu einem angemessenen Verständnis dieses Problems wäre wohl die Aufgabe, das, was sich uns im Alltagsverständnis als "Anspruchsniveau» darstellt, als Kovariation einer Reihe von gesellschaftlich wirksamen Faktoren, die zu einem bestimmten individuellen Verhalten führen, zu begreifen. Heißt dies, daß es überhaupt keine menschnaturhaft-persönlichen Bestimmungsmomente im Verhalten der Individuen gibt? Lassen wir die Frage ruhig unbeantwortet stehen; ihre Lösung erscheint im übrigen als ein praktisches und kein theoretisches Problem, wie uns die Verhaltensexperimente mit Menschen - indem man sie z. B. in Isolationshaft bringt - zu beweisen versuchen. Ein grundlegender Fehler wäre nur - falls es solche Bestimmungsmomente gibt - , sie frei von einer Formbestimmtheit durch die jeweiligen gesellschaftlichen Verhältnisse anzusehen. Veranschaulichen wir uns diesen Sachverhalt wiederum an einem Beispiel:

Bei der Untersuchung des Einkaufsverhaltens, etwa in bezug auf den alltäglichen Lebensmittelbedarf, stößt man sicher sehr bald auf das Phänomen, daß eine Gruppe von Konsumenten ihre Einkäufe häufiger als andere Konsumenten beim "Kaufmann an der Ecke» tätigt. Nach den Gründen für dieses spezielle «räumliche Verhalten» befragt, geben nicht wenige Vertreter dieser Gruppe, vor allem ältere und alleinstehende Menschen, an, daß sie diesen Kaufmann als einen «freundlichen, immer zu einem kleinen - wenn auch manchmal kurzen - Gespräch bereiten Menschen» kennen, und daß man die "persönliche Atmosphäre» dieses Ladens, der «bekanntlich sogar teurer als der nahe Supermarkt» ist, der «Anonymität» des Einkaufens in dem Großgeschäft vorziehe. - Ich will dieses Beispiel nicht weiter ausführen; man kann es auch sicherlich viel differenzierter behandeln. Worauf es mir ankommt, ist zu zeigen, daß die "zwischenmenschlichen Beziehungen» zwischen dem Ladenbesitzer und den Konsumenten, deren räumliches Verhalten sie zu erklären scheinen, nicht auf naturhaft-persönliche Einstellungen oder bloße Situationsbedingtheiten reduzierbar sind. Die eigentliche Schwierigkeit dabei ist, die konkret-historische Formbestimmtheit des Verhaltens der Beteiligten nicht nur fallweise-empirisch, sondern allgemein-theoretisch einzuholen, ohne den Fehler der behavioristischen oder aktionistischen Verhaltenstheorie zu wiederholen. So müßte aufgezeigt werden, inwiefern «Freundlichkeit» von Verkäufern scheinpersönliches "Verhalten gegenüber der Umwelt» ist, weil verbunden mit dem Interesse an der Hebung des Warenumsatzes, ein Verhalten, 
das dem Ladenbesitzer durch die Konkurrenz der vielen Einzelkapitale um höchstmöglichen Profit aufgezwungen wird (bei Strafe des Untergangs, wenn er einen bestimmten Umsatz nicht erzielt). Weiter wäre theoretisch zu begründen, inwiefern die Wahlhandlungen einer bestimmten Gruppe von Käufern Ausdruck des Scheins echter «Handlungsalternativen im Raum» einer warenproduzierenden und warentauschenden Gesellschaft ist. Dieser je bezügliche Schein stellt allerdings nichts Imaginäres dar, sondern er ist real in dem Sinne, daß er durch die objektiven Verhältnisse bedingt wird, also durchaus Teil der gesellschaftlichen Realität ist. Der empiristische Verhaltenstheoretiker verstellt und verkehrt diesen (gesellschaftlichen) Zusammenhang von objektiver Wirklichkeit, realem Schein und subjektivem Bewußtsein, indem er das Verhalten der Menschen entweder aus allgemeinen menschlichen Dispositionen oder aus unmittelbaren situativen Bedingungen «erklärt».

Die Konsequenz, die aus diesen Ausführungen für eine nicht-empiristische Gesellschaftstheorie gezogen werden müßte, wäre zumindest eine doppelte. Zum einen müßte eine solche Theorie eine Begrifflichkeit beinhalten, deren Charakter "objektiv» und «subjektiv» zugleich wäre, indem diese sich gleichermaßen auf das Verhalten der Individuen wie auf die gesellschaftlichen Verhältnisse beziehen ließe ${ }^{23)}$. Zum zweiten würde eine solche Theorie ein anderes Interesse am Menschen verfolgen, als es die verhaltenswissenschaftliche Forschung mit ihrem Interesse an der Prognostizierbarkeit und Steuerbarkeit menschlichen Verhaltens zeigt, nämlich: sie müßte sich bemühen aufzuzeigen, daß das Bewußtsein der Menschen, indem es den realen Schein reproduziert, «entfremdetes» Bewußtsein darstellt, weil es nicht mehr Selbst-Bewußtsein, d. h. Bewußtsein gerade jener Verhältnisse ist, in denen die Menschen zu bloßen Vollzugsorganen verkümmern. Auch wäre die Unterordnung der Individuen unter schon als allgemein gesetzte Bedingungen des Verhaltens nicht mehr als problemlose Ausgangsfeststellung bei der Untersuchung der Handlungsspielräume der Menschen anzusehen, sondern mit der je spezifischen «Rationalität» einer Gesellschaftsordnung zu vermitteln. So bewirkt z. B. im Kapitalismus die spezifische Rationalität der Profitmaximierung eine - wie MAUKE treffend formuliert ${ }^{24)}$ - «Funktionalisierung der Menschen zu (Agenten) der als sachliche Agentien verselbständigten Produktionsverhältnisse». Diese Verhältnisse ermöglichen darüber hinaus sowohl auf der Seite der Herrschenden wie auf der Seite der Beherrschten eine wachsende Auswechselbarkeit der Menschen. Diese erscheinen dann als unhistorische Wesen mit verschiedenen ihnen anhängenden «invarianten» Verhaltens-Eigenschaften, quasi als Mensch-Korpuskeln, deren unterschiedliches Agieren und Reagieren vom Verhaltensforscher wahlweise-beliebig zu «Gruppen gleichen (räumlichen) Verhaltens» aggregiert werden kann. Garantiert wird dies durch eine "Methode», und realisiert wird es durch «Empirie». Daß dies überhaupt möglich ist, begründet sich dadurch, daß es sich bei den «Verhaltens-Gesetzmäßigkeiten» und bei den "Verhaltens-Gruppen» nicht um bloß gedachte, sondern um reale Determinationen bzw. Divisionen der Menschen unter bestimmten gesellschaftlichen Verhältnissen handelt. Dieser Sachverhalt wird weder von der behavioristischen noch von der aktionistischen Verhaltensforschung in den methodischtheoretischen Ansatz einbezogen. Darin allgemein liegt ihre Funktionalität für Zwecke der herrschenden Interessen, nicht erst in der Manipulation für besondere Zwecke.

\section{Anmerkungen}

1) Vgl.zur «Logik» dieser Veränderung EISEL 1980, insb. Kap. 3, S. 185 ff., sowie Kap. 5.2, S. 517 ff.

2) Bei diesen handelt es sich um die Wiederaufnahme und Weiterführung meiner Darlegungen 1981 a und 1981b. In bezug auf die verwendete Literatur kann ich mich deshalb an dieser Stelle auf die unmittelbar oder zusätzlich zitierte Literatur beschränken.

3) Eine solche Trennung bzw. Gegenüberstellung beinhaltet immer auch eine gewisse Problematik (vgl. etwa GRAUMANN 1980). Dadurch werden z. B. Differenzierungen, wie sie innerhalb dieser Ansätze - so zwischen (neuerer) Einstellungsforschung und (herkömmlichem) Behaviorismus - gesehen werden (s. MEINEFELD 1977, S. 206), und die in einem anderen als dem hier behandelten Zusammenhang durchaus Bedeutung erlangen, überdeckt. Auch könnte man mit guten Gründen Teile der Aktionsforschung als "gemäßigten» (oder: "methodologischen") Behaviorismus bezeichnen, der den Bereich der menschlichen Einstellungen lediglich wegen seiner mangelnden Überprüfbarkeit ausschaltet. Oder man könnte, wie HABERMAS (1970, S. 138 ff.), zwischen einer generellen Verhaltenswissenschaft - dem "behavioristischen Ansatz" - und einer (spezielleren; G. B.) Theorie des Handelns - dem «aktionistischen Ansatz» - unterscheiden.

4) Vgl. MEINEFELD 1977, S. 26. - Der Ansatz geht also über die Wahrnehmungsgeographie i.e.S. ("perceptual geography») hinaus.

5) Vgl. THOMALE 1974, S. 14 ff.; WIESSNER 1978, S. 421.

6) THOMALE 1974, S. 14.

7) Siehe Anm. 5.

8) Vgl. hierzu etwa die Vorträge der Fachsitzung «Aktionsräumliche Ansätze in der Zentralitätsforschung» auf dem 42. Dt. Geographentag Göttingen 1979 (DEITERS/HEINRITZ 1980); diese Referate sind in vollständiger Fassung erschienen in der Geographischen Zeitschrift 67 (1979), H. 4.

9) Vgl. CORNFORTH 1970, S. 19.

10) Eine weitergehende Einschätzung gibt HARD 1973, S. $211 \mathrm{ff}$.

11) Siehe HÖLLHUBER 1977, S. $27 \mathrm{ff}$.

12) Vgl. auch HOLZKAMP 1972, S. $105 \mathrm{f}$.

13) Siehe KASTER/LAMMERS 1979, passim.

14) BRUDER 1972, S. 95.

15) In dem zitierten Beispiel (WOLFFERT 1970) geht der Autor so vor.

16) GREIFF 1976, S. 17, Anm. 13.

17) Ibid.

18) BRUDER 1973, S. 98; Hervorhebung G. B.

19) Vgl. die Belege in BECK 1981b, Anm. 13.

20) Vgl. EISEL 1980, S. 12. 
21) Vgl. etwa den in BECK 1981 a, Anm. 9, zitierten Beleg.

22) Zur allgemeinen Wahrscheinlichkeit der "social career» einer sozialwissenschaftlichen Theorie vgl. GOULDNER 1974, Bd. 1, insb. S. 40f. - Ähnliches gilt wohl in bezug auf den Wechsel der Hintergrundannahmen von sozialwissenschaftlichen Theorien ("Paradigmenwechsel»).

23) Vgl. dazu etwa die Diskussion zwischen PARIS 1976 und OTTOMEYER 1976. - Ich sollte wohl wenigstens ein Beispiel einer empirischen Untersuchung angeben, welche in der Tendenz einem solchen theoretischen Ansatz entspricht: Man prüfe diesbezüglich HARVEY 1974, eine Untersuchung, in welcher der Autor mit Begriffen wie "Transmissionsagent", "objektive/subjektive Klassenlage» usw. arbeitet.

24) MAUKE 1971, S. 80.

\section{Literatur}

BECK, G., 1981 a: Darstellung und Kritik des verhaltens- und entscheidungstheoretischen Ansatzes in der Geographie. In: OSTHEIDER, M./STEINER, D. (Hg.): Theorie und Quantitative Methodik in der Geographie. Symposium, Zürich 1980. (Zürcher Geogr. Schriften; 1) Zürich, S. 119-139.

BECK, G. 1981 b: Der verhaltens- und entscheidungstheoretische Ansatz. Zur Kritik eines modernen Paradigmas in der Geographie. In: SEDLACEK, P. (Hg.): Kultur-/Sozialgeographie. Beiträge zu ihrer wissenschaftstheoretischen Grundlegung. (Uni-Taschenbücher; UTB 1053) Paderborn (im Druck).

BRUDER, K.-J., 1972: Verhalten als Funktion der Bedingungen von Verhalten. Zum Doppelcharakter der bürgerlichen Psychologie. In: BRINKMANN, H./BRUDER, K.-J./MÜNCH, R.: Wissenschaftstheorie + Gesellschaftliche Praxis. (edition 2000, theorie + kritik; 3) Gießen, S. 81-102.

BRUDER, K.-J., 1973: Entwurf der Kritik der bürgerlichen Psychologie. In: Ders. (Hg.): Kritik der bürgerlichen Psychologie. Zur Theorie des Individuums in der kapitalistischen Gesellschaft. (Bücher d. Wissens; Fischer-Tb. 6198) Frankfurt a. M., S. 92-217.

CORNFORTH, M., 1970: Marxistische Wissenschaft und antimarxistisches Dogma. (Marxist. Taschenbücher) Frankfurt a. M.

DEITERS, J./HEINRITZ, G. (Leitung), 1980: Fachsitzung «Aktionsräumliche Ansätze in der Zentralitätsforschung». In: SANDNER, G./NUHN, H. (Hg.): 42. Deutscher Geographentag Göttingen 1979. Tagungsbericht und wissenschaftliche Abhandlungen. (Verh.d. Dt. Geogr.-Tages; 42) Wiesbaden, S. 427-441.

EISEL, U., 1980: Die Entwicklung der Anthropogeographie von einer «Raumwissenschaft» zur Gesellschaftswissenschaft. (Urbs et Regio, Kasseler Schriften z. Geografie u. Planung; 17) Kassel.

GOULDNER, A. W., 1974: Die westliche Soziologie in der Krise. 2 Bde. (rowohlts dt. enzyklopädie; 360/361) Reinbek b. Hamburg. GRAUMANN, C. F., 1980: Verhalten und Handeln - Probleme einer Unterscheidung. In: SCHLUCHTER, W. (Hg.): Verhalten,
Handeln und System. Talcott Parsons' Beitrag zur Entwicklung der Sozialwissenschaften. (suhrkamp taschenbuch wissenschaft; 310) Frankfurt a. M., S. 16-31.

GREIFF, B.v., 1976: Gesellschaftsform und Erkenntnisform. Zum Zusammenhang von wissenschaftlicher Erfahrung und gesellschaftlicher Entwicklung. (Campus Diskussion: Krit. Sozialwiss.) Frankfurt/M., New York.

HABERMAS, J., 1970: Ein Literaturbericht (1967): Zur Logik der Sozialwissenschaften. In: Ders.: Zur Logik der Sozialwissenschaften. (ed. suhrkamp; 481) Frankfurt a. M., S. 71-310.

HARD, G., 1973: Die Geographie. Eine wissenschaftstheoretische Einführung. (Sammlung Göschen; 9001) Berlin, New York.

HARVEY, D., 1974: Klassenmonopolrente, Finanzkapital und Urbanisierung. In: Stadtbauwelt 41, S. 25-34. Wieder abgedruckt in MAYER, M./ROTH, R./BRANDES, V. (Hg.): Stadtkrise und soziale Bewegungen. Texte zur internationalen Entwicklung. (Polit. Ökonomie. Geschichte u. Kritik) Köln 1978, S. 55-77.

HÖLLHUBER, D., 1977: Modelle des wirtschaftenden Menschen in der Geographie. In: Wirtschaftsgeogr. Studien 1, S. 17-36.

HOLZKAMP, K., 1972: Kritische Psychologie. Vorbereitende Arbeiten. (Texte z. polit. Theorie u. Praxis; Fischer-Tb. 6505) Frankfurt a.M.

KASTER, T./LAMMERS, D. A., 1979: Ausgewählte Materialien zur Zeitgeographie. (Karlsruher Manuskripte z. Mathemat. u. Theoret. Wirtschafts- u. Sozialgeogr.; 35) Karlsruhe.

MÅRTENSSON, S., 1979: Der Einfluß regionaler Disparitäten auf die Zeitbudgets ausgewählter Haushaltstypen. In: KASTER/ LAMMERS 1979, S. 34-67.

MAUKE, M., 1971: Die Klassentheorie von Marx und Engels. (Krit. Studien z. Politikwiss.) 3.A. Frankfurt a. M.

MEINEFELD, W., 1977: Einstellung und soziales Handeln. (rororo studium; 103) Reinbek b. Hamburg.

OTTOMEYER, K., 1976: Antikritische Bemerkungen zu Rainer Paris. In: Gesellschaft (Hg.): Beiträge zur Marxschen Theorie 8/9. (ed. suhrkamp; 863) Frankfurt a. M., S. 335-349.

PARIS, R., 1976: Schwierigkeiten einer marxistischen Interaktionstheorie. Anmerkungen zu einem Programm der Vermittlung von Kritik der politischen Ökonomie und Interaktionstheorie. In Gesellschaft (Hg.): Beiträge zur Marxschen Theorie 7. (ed.suhrkamp; 827) Frankfurt a. M., S. 11-44.

THOMALE, E., 1974: Geographische Verhaltensforschung. In: DICKEL, H., u.a.: Studenten in Marburg. (Marburger Geogr. Schriften; 61) Marburg/Lahn, S. 9-30.

WIESSNER, R., 1978: Verhaltensorientierte Geographie. Die angelsächsische behavioral geography und ihre sozialgeographischen Ansätze. In: Geogr. Rundschau 30, 1978, S. 420-426.

WOLPERT, J., 1970: Eine räumliche Analyse des Entscheidungsverhaltens in der mittelschwedischen Landwirtschaft. In: BARTELS, D. (Hg.): Wirtschafts- und Sozialgeographie. (Neue Wiss. Bibliothek; 35) Köln, Berlin, S. 380-387.

\section{Diskussion zum Vortrag Günther Beck}

Diskussionsteilnehmer:

GB Dr. GÜNTHER BECK, Geograph, Göttingen (Referent)

KI Dr. KLAUS I. ITTEN, Geograph, Zürich (Diskussionsleiter)

PB PETER BƯNZLI, Geographiestudent, Zürich

PH Dr. PAUL HOYNINGEN, Wissenschaftsphilosoph, Zürich HW HERBERT WANNER, Geograph, Zürich

KI: Als Eröffnung der Diskussion möchte ich Ihnen folgende Frage stellen: In unserem Seminar zur Theorie in der Geographie haben wir in der letzten Sitzung über den Raumbegriff in der Geographie gesprochen. Welches ist nun Ihr Raumbegriff in der Verhaltensgeographie?
GB: Im behavioristischen Ansatz steht «Raum» gleichbedeutend für die Gesamtheit derjenigen Umweltgegebenheiten, die vom Individuum sinnlich wahrgenommen werden. Die Aufzeichnung solcher "Wahrnehmungsräume» ergibt jene in der Regel stark verzerrten Landschaftsbilder, wie sie in den bekannten "mental maps» der Perzeptionsforscher dargestellt sind. Im aktionistischen Ansatz bezeichnet «Raum» die Gesamtheit derjenigen Umweltgegebenheiten, die die "Bewegungs(spiel)räume» der Individuen bilden. Die Abbildungen solcher Räume sind von der Art der geometrischen Gebilde, wie sie 